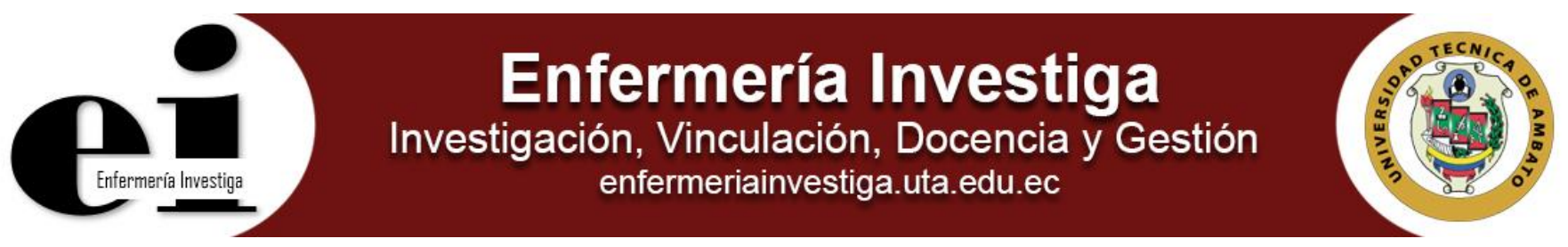

DOI: http://dx.doi.org/10.29033/ei.v3n2.2018.05

Artículo original

\title{
Factores maternos que inciden en amenaza de parto pretérmino en adolescentes gestantes del cantón San Jacinto de Yaguachi
}

Maternal factors that affect the threat of preterm delivery in pregnant adolescents of San Jacinto de Yaguachi canton

\section{Joicy Anabel Franco Coffre ${ }^{1}$}

${ }^{1}$ Universidad de Guayaquil - Facultad de Ciencias Médicas - Carrera de Enfermería - Guayaquil - Ecuador

Franco JA. Caracterización clínico e histopatológica de pacientes con tumor de conjuntiva. Enferm Inv. 2018;3(2):79-84.

2477-9172 / 2550-6692 Derechos Reservados @ 2018 Universidad Técnica de Ambato, Carrera de Enfermería. Este es un artículo de acceso abierto distribuido bajo los términos de la Licencia Creative Commons, que permite uso ilimitado, distribución y reproducción en cualquier medio, siempre que la obra original es debidamente citada.

\section{Historia:}

Recibido: 20 febrero 2018

Revisado:30 abril 2017

Aceptado: 2 julio 2018

Palabras Claves: Trabajo de parto prematuro; embarazo en adolescencia; rotura prematura de membranas fetales;

promoción de la salud; enfermería materno infantil

Keywords: Premature labor; adolescent pregnancy; premature rupture of fetal membranes; health promotion; maternal and child nursing

\section{Resumen}

Introducción: La amenaza de parto pre término consiste en la presencia de contracciones regulares después de las 20 y antes de las 37 semanas de gestación Objetivo: Determinar los factores maternos que inciden la amenaza de parto pretérmino en las mujeres gestantes que acuden al Hospital Básico de Yaguachi.

Métodos: Sé realizo un Estudio cuantitativo, descriptivo, y transversal en el Hospital Básico "Dr. José Cevallos Ruiz", con una muestra de 20 adolescentes de sexo femenino, con el diagnostico amenaza de parto pretérmino, a las que se les aplicó una encuesta que constó con 5 preguntas entre ellas: Edad, educación, domicilio, controles prenatales y patología asociada a su embarazo.

Resultados: Predominó el grupo de adolescentes correspondiente a los 17- 19 años con un 65\%, instrucción secundaria el $55 \%$, el $80 \%$ de las gestantes residía en la zona rural. En cuanto a los controles prenatales el $60 \%$ de las adolescentes asistieron de 1 a 4 controles, y el $50 \%$ de las adolescentes presentaron como factor de riesgo para la amenaza de parto pretérmino, la rotura prematura de membranas como patología asociada a su embarazo.

Conclusiones: El factor materno que predominó fue la rotura prematura de membranas, sin embargo la infección de vías urinarias y los trastornos hipertensivos del embarazo, también se encontraron presentes y por ello, la educación prenatal debe ser tomada en gran consideración.

\section{Abstract}

Introduction: The threat of preterm birth consists in the presence of regular contractions after 20 and before 37 weeks of gestation. Objective: To determine the maternal factors that affect the threat of preterm delivery in pregnant women who come to the Basic Hospital of Yaguachi

Methods: A quantitative, descriptive, and transversal study in the Basic Hospital "Dr. José Cevallos Ruiz ", with a sample of 20 female adolescents, with the diagnostic threat of preterm delivery, to which a survey was applied that consisted of 5 questions including: Age, education, home, prenatal check-ups and associated pathology to your pregnancy.

Results: The group of adolescents corresponding to $17-19$ years old predominated with $65 \%$, secondary instruction 55\%, $80 \%$ of pregnant women resided in the rural area. Regarding the prenatal controls, $60 \%$ of the adolescents attended from 1 to 4 controls, and $50 \%$ of the adolescents presented as a risk factor for the threat of preterm delivery, the premature rupture of the membranes as a pathology associated with their pregnancy.

Conclusions: The maternal factor that predominated was the premature rupture of membranes, nevertheless the infection of urinary tract and the hypertensive disorders of pregnancy, were also present and therefore, prenatal education should be taken into great consideration

Autor de correspondencia:

Joicy Anabel Franco Coffre. Carrera de Enfermería - Facultad de Ciencias Médicas - Universidad de Guayaquil - Guayaquil - Ecuador. Teléfono: +593986392909. E-mail: anniafrank18@gmail.com 


\section{Introducción}

El embarazo pretérmino se define como la presencia de contracciones con un ritmo de 4 cada 20 minutos o de 8 en 60 minutos luego de las 20 semanas de gestación y antes de las 37 semanas Este hecho se debe asociar al menos a una de las siguientes circunstancias: Modificación progresiva del cérvix: dilatación cervical > a $2 \mathrm{~cm}$ y borramiento $>80 \%{ }^{1}$

Todos los años nacen en el mundo alrededor de 13 millones de niños prematuros. ${ }^{2}$ El parto prematuro está asociado como la principal causa de morbimortalidad neonatal, manifestando múltiples factores que comprometen a la madre entre ellos; socioeconómicos y culturales, complicaciones médicas y cuidados prenatales deficientes, mujeres solteras o sin apoyo social, con adicciones, 0 también portadoras de factores genéticos, ${ }^{3}$ y del mismo modo la presencia de factores que comprometan al feto entre ellos tenemos: las malformaciones congénitas, restricción del crecimiento intrauterino y muertes fetales.

La mayoría de los embarazos en adolescentes no son planificados. Las consecuencias del embarazo precoz y no planificado son varias. Las adolescentes embarazadas son más propensas a retrasar la búsqueda de atención prenatal y tienen mayores tasas de resultados de nacimiento desfavorables, como la prematuridad, la mortalidad infantil $y$ retrasos en el desarrollo. Además, las madres adolescentes tienen más probabilidades de abandonar la escuela, enfrentar el desempleo, vivir en la pobreza y depender de la asistencia pública que sus pares que no tienen hijos. Debido a la falta de orientación en los jóvenes, se observa cada día un incremento en el número de adolescentes embarazadas, la actividad sexual ocurre de forma mas temprana. ${ }^{4}$ Su frecuencia varía de 5 a $11 \%$ en las regiones desarrolladas y hasta $40 \%$ en algunas regiones muy pobres. Sin embargo las más afectada la zona rural con un índice elevado del $60 \%{ }^{5}$

Existe evidencia insuficiente acerca de la efectividad del uso de índices de riesgo poblacionales enfocadas en la reducción de la incidencia del parto pretérmino. No se discrimina a las mujeres que se verían beneficiadas con programas de prevención y las que no. Sin embargo aquellas pacientes que presentaron un parto pretérmino previamente, tienen alta posibilidad de recurrencia estimada entre 17 y $37 \% .{ }^{6}$ Se relaciona a la aparición de la amenaza de parto pretérmino: infecciones de vías urinarias (IVU), estado nutricional deficiente a sus necesidades, trastornos hipertensivos del embarazo, anemia, rotura prematura de membranas, consumo de sustancias psicotrópicas, entre otras.

Fisiopatológicamente se cataloga como un incremento de la contractibilidad miometrial, cambios cervicales o cambios en la consistencia del útero y activación de la decidua en las membranas corioamnióticas. ${ }^{7}$ La amenaza de parto pretérmino generalmente se ve ligado a distintos tipos de anomalías en la mujer gestante y se relaciona con rotura prematura de membranas, placenta previa, casos también en los que se da espontáneamente sin etiología alguna ósea de origen idiopático. ${ }^{2-6}$

Las opciones diagnósticas para prevenir y detectar a tiempo las amenazas de parto pretérmino ayudaran al médico mediante el líquido amniótico que recibirá el nombre de fibronectina fetal, el cual actúa como un indicador para que el medico inicie con un tratamiento más enérgico y así evitar oportunamente labores de parto pretérmino. ${ }^{7}$

Mediante cuidados oportunos el personal de enfermería intervendrá en el tratamiento de una manera oportuna y eficaz, realizando cuidados óptimos para el bienestar integral como; reposo absoluto, hidratación, uso de agentes tocolíticos (bajo prescripción médica si resultara necesario para la paciente) y estimulación de la madurez pulmonar fetal. ${ }^{6-7}$

El cantón San Yaguachi cuanta con 4 parroquias representando el $48.7 \%$ de la población femenina, evidenciando el hábitat con el $70.8 \%$ en zona rural y $29.2 \%$ en zona urbana. ${ }^{8}$ Entre los factores que determinan la problemática de la amenaza de parto pretérmino, se encuentra la edad. Se ha demostrado que las madres adolescentes tienen más probabilidades de presentar comportamientos desfavorables durante el embarazo, que las mujeres que ya han cumplido su mayoría de edad. Por otro lado, el nivel de escolaridad como factor predisponente en esta complicación del embarazo, se relaciona con las actitudes y prácticas de las adolescentes con respecto al cuidado de salud y percepción de riesgos. ${ }^{9}$

Oportunamente el profesional de enfermería desempeña un papel muy importante, brindando cuidados holísticos y humanos con responsabilidad, y así obtener en menor tiempo posible mejorías mediante intervenciones especiales e individualizadas en cada paciente que atraviese dificultades en su salud, manifestando apoyo, confort y manteniendo una relación optima entre enfermerapaciente y familia del paciente inculcando la promoción del cuidado. ${ }^{10}$

Nola Pender, enfermera, autora del Modelo de Promoción de la Salud (MPS), identifica conceptos relevantes sobre las conductas de promoción de la salud e integra los hallazgos de investigación referente al individuo de tal manera que este proceso facilite la generación de hipótesis comparables. ${ }^{11}$ Esta teoría continua siendo perfeccionada y ampliada en cuanto su capacidad para explicar las relaciones entre los factores que influyen. ${ }^{12} \mathrm{EI}$ objetivo de esta teoría es motivar en las personas el deseo de alcanzar el óptimo bienestar, basándose en la educación de las personas sobre cómo cuidarse y promover estilos de vida saludable, esto es primordial, mucho más que los cuidados, porque de 
ese modo hay menos gente enferma. ${ }^{11-12}$ Esta teoría está asociada a la amenaza de parto pretérmino mediante la promoción y prevención que el profesional de enfermería brindase a través de la educación en la atención primaria, resaltando así la importancia de los controles prenatales e incentivando al cuidado propio y evitar posibles complicaciones durante el periodo de gestación.

\section{Materiales y métodos}

El presente estudio fue de naturaleza cuantitativa y de tipo descriptivo, con corte transversal. Se realizó en el Cantón San Jacinto de Yaguachi de la provincia del Guayas-Ecuador, en el Hospital Básico "Dr. José Cevallos Ruiz", se seleccionaron a 20 mujeres primíparas adolecentes en estado de gestación con amenaza de parto pretérmino, con edades gestacionales entre 28 y 36 semanas. Se incluyó a todas las adolescentes entre el rango de 10 -19 años de edad, atendidas en el Hospital Básico de Yaguachi, en el mes de Enero del 2018. Se excluyeron a todas aquellas adolescentes que no presentaron amenaza de parto pretérmino, así como aquellas embarazadas con amenaza de parto pretérmino en franco trabajo de parto. Como parte de la ética del estudio se explicó e hizo firmar el consentimiento informado para determinar los indicadores de hechos manifestados y observables en adolescentes con amenaza de parto pretérmino.

Se aplicó una encuesta que incluyo; edad, nivel de educación, zona domiciliaria, controles prenatales, manifestaciones durante el embarazo y morbilidades en el periodo de embarazo. La recolección de datos fue no experimental con modalidad de campo, utilizando la técnica de recolección de datos mediante encuestas aplicadas a las adolescentes atendidas en el periodo anteriormente citado. Efectuándose de manera anónima para garantizar la autonomía, privacidad y no maleficencia hacia este grupo de mujeres, recibiendo colaboración de cada una de ellas.

\section{Resultados}

Tabla 1: Distribución de adolescentes según su rango de edad.

\begin{tabular}{lcc}
\hline Edad & Frecuencia & $\%$ \\
$10-13$ años & 1 & 5 \\
$14-16$ años & 6 & 30 \\
$17-19$ años & 13 & 65 \\
\hline $\begin{array}{c}\text { Total } \\
\text { Fuente: Encuestas realizadas a adolescentes del Hospital Básico "Dr. José } \\
\text { Cevallos Ruiz". }\end{array}$
\end{tabular}

Se observó que en el Hospital Básico "Dr. José Cevallos Ruiz", existió preponderancia de las adolescentes con edades entre 17 a 19 años representadas por el 65\%. Las adolescentes con edades inferiores a 13 años resultaron las menos frecuentes $(5 \%)$.

Tabla 2: Distribución de adolescentes según su nivel educativo.

\begin{tabular}{ccc}
\hline Edad & Frecuencia & $\%$ \\
Ninguno & 3 & 15 \\
Primaria & 2 & 10 \\
Secundaria & 11 & 55 \\
Nivel superior & 4 & 20 \\
\hline Total & 20 & 100 \\
Fuente: Encuestas realizadas a adolescentes del Hospital Básico "Dr. José \\
\end{tabular}

En la tabla se observa el predominio de la instrucción académica secundaria correspondiente al $55 \%$, y en un $10 \%$ correspondieron a la instrucción primaria.

Tabla 3: Distribución de adolescentes según su ubicación del domicilio.

\begin{tabular}{lcc}
\hline Ubicación & Frecuencia & $\%$ \\
Rural & 16 & 80 \\
Urbano & 4 & 20 \\
\hline \begin{tabular}{l} 
Total \\
Fuente: Encuestas realizadas a adolescentes del Hospital Básico "Dr. José \\
\multicolumn{2}{c}{ Cevallos Ruiz". }
\end{tabular}
\end{tabular}

En cuanto a la ubicación del domicilio, el $80 \%$ de las adolescentes con amenaza de parto pretérmino residían en el área rural del cantón Yaguachi.

Tabla 4: Distribución de adolescentes según los controles prenatales.

\begin{tabular}{lcc}
\hline Ubicación & Frecuencia & $\%$ \\
1 a 4 & 12 & 60 \\
$>5$ & 8 & 40 \\
\hline $\begin{array}{c}\text { Total } \\
\text { Fuente: Encuestas realizadas a adolescentes del Hospital Básico "Dr. José } \\
\end{array}$
\end{tabular}

Con relación a los controles prenatales, el $60 \%$ de mujeres tuvieron de 1 a 4 controles, lo cual es considerado como un buen control durante el embarazo.

Tabla 5: Patologías asociadas a la amenaza de parto pretérmino. 


\begin{tabular}{ccc}
\hline $\begin{array}{c}\text { Patologías } \\
\text { Infección de } \\
\text { vías urinarias } \\
\text { Anemia }\end{array}$ & Frecuencia & $\%$ \\
$\begin{array}{c}\text { Rotura } \\
\text { prematura de } \\
\text { membrana } \\
\begin{array}{c}\text { Placenta } \\
\text { Previa }\end{array}\end{array}$ & 10 & 20 \\
$\begin{array}{c}\text { Trastornos } \\
\text { Hipertensivos }\end{array}$ & 2 & 50 \\
\hline $\begin{array}{c}\text { Total } \\
\text { Fuente: Encuestas realizadas a adolescentes del Hospital Básico "Dr. José } \\
\text { Cevallos Ruiz". }\end{array}$ & 10 \\
& 3 & 15 \\
& & 100
\end{tabular}

La principal patología asociada a la amenaza de parto pretérmino de las adolescentes embarazadas del cantón Yaguachi correspondió al diagnóstico de rotura prematura de membranas, y en menor instancia la anemia con el $5 \%$ de incidencia.

\section{Discusión}

Se determinó que el $65 \%$ de las adolescentes de la muestra de estudio, correspondieron a la etapa de la adolescencia tardía, esto se traduce a un comienzo precoz de las relaciones sexuales, mucho antes de cumplir su mayoría de edad. El enfrentarse a un embarazo donde cambios fisiológicos importantes aparecen en el cuerpo de la mujer, trastoca a la adolescente a sufrir un impacto importante en su ser, tanto físicamente (ya que los órganos de reproducción femenino no se encuentran totalmente desarrollados) como también en sus aspectos psicológico y social. En un estudio realizado en Lima, Perú en el Hospital IV "Augusto Hernández Mendoza" la amenaza de parto pretérmino en las adolescentes, apareció en edades entre los 15 - 19 años. ${ }^{13}$ En el caso de Ecuador esta problemática se presenta en un 19\% de embarazos en adolescentes, mientras que en el Perú tienen un $13 \%$ de embarazos esta fase etaria. ${ }^{14}$

La zona rural del Cantón Yaguachi es vasta. Existen caminos vecinales, con pasos peatonales improvisados e inseguros, dificultades en el acceso automotor, o inclusive, en ciertos recintos la única forma de ingreso es a pie o en moto. Tomando en cuenta que las zonas rurales tienen mayor desventaja con respecto a los cuidados de la salud, la mujer en estado de gestación, omite los controles prenatales o tiende a esperar a que llegue el momento del parto para poder acudir al médico. Dentro de los factores de riesgo para el desarrollo de la amenaza de parto pretérmino se declara justamente que los factores socioeconómicos, el nivel educacional, y los domicilios ubicados en áreas aisladas podrían determinar la incidencia de esta patología. ${ }^{15}$
Según la OMS, concluyo a nivel mundial que los controles de embarazos de bajo riesgo podrían ser $4 .{ }^{16}$ Mientras que los reportes de la investigación demuestran que las adolescentes de alto riesgo con amenaza de parto pretérmino no acuden a los controles convirtiéndose en una problemática de salud para las adolescentes, conforme así las patologías asociadas al parto pretérmino es la principal causa de morbilidad y mortalidad perinatal. ${ }^{17}$

La rotura prematura de membranas aquí descrita como primer factor materno de la amenaza de parto pretérmino posee como factores de riesgo: los factores fisiológicos, genéticos y ambientales maternos. Estos factores de riesgo son similares a los del trabajo de parto prematuro, pero la mayoría de los pacientes no tienen factores de riesgo identificables. ${ }^{18}$ En un estudio realizado en Inglaterra durante el año 2007, en 4591 embarazos, obtuvieron como conclusión principal que las probabilidades de partos prematuros ( $<32$ semanas) y extremadamente prematuros (<28 semanas) son mayores en el grupo de embarazadas adolescentes. ${ }^{19}$

El segundo factor que está comprometido en la amenaza de parto pretérmino son las infecciones de vías urinarias (IVU). En referencia a un estudio realizado con la misma temática en la Maternidad Mariana de Jesús de la ciudad de Guayaquil, cuya muestra incluyó 100 adolescentes en estado de gestación y cuya finalidad fue relacionar la infección de vías urinarias como factor de riesgo de la amenaza de parto pretérmino, concluyeron que 60 casos $(60 \%)$ de las adolescentes que tenían infección de vías urinarias progresaron a amenaza de parto pretérmino, adicionalmente, de éstos casos el $50 \%$ tuvo esta complicación dentro de las semanas 34 - 36 de gestación y por grupos de edad, las pacientes de 17 a 19 años son las que más incidencia tuvieron. ${ }^{20}$ En el caso Yaguachi, las infecciones de vías urinarias se encontraron en un $20 \%$ asociadas a la amenaza de parto pretérmino contrastando con el resultado de la Maternidad Mariana de Jesús.

El embarazo en adolescentes puede tener consecuencias adversas para la salud en corto como a largo plazo tanto para la madre adolescente y los neonatos, esto conlleva a riesgos de mal formaciones congénitas, síndrome de dificultad respiratoria, ${ }^{21}$ patologías infecciosas, y entre otras. ${ }^{22,}$ 24 Ante la alarmante situación de embarazos en adolescentes especialmente en el Cantón Yaguachi, existe la necesidad de llevar a cabo políticas eficaces, eficientes y efectivas para contrarrestar el índice elevado de patologías asociadas con el embarazo a temprana edad.

\section{Conclusiones}

La Rotura prematura de membranas fue una de las causas, mas prevalentes para la amenaza de parto pretermino en las adolescentes embarazadas, sin embargo la infeccion de vias urinarias y los 
trastornos hipertensivos de embarazo fueron tambien, en menor porcentaje, el origen de esta complicacion. En todas las causas aqui enunciadas, se observa que en la mayoria de casos son situaciones prevenibles y que pueden ser evitadas. Sin embargo, la adolescencia representa un estado de inmadurez tanto fisica como mental que se vuelve tambien, un factor de riesgo importante que atenta al embarazo y que por ende pone en riesgo la vida del feto y la madre.

Los controles prenatales son de notable trascendencia para que este embarazo que de por si, es un embarazo de alto riesgo, llegue a un feliz termino. Evitar complicaciones, detectar patologias concominantes, evaluar estado nutricional, crecimiento fetal y demas, son cuidados que obtiene la adolescente embarazada al acudir a su control prenatal. La familia, la comunidad y el servicio de salud que le atañe estas jovenes, tiene un duro camino que recorrer brindandoles apoyo, educacion y no estigmatizacion y mas bien creando espacios amigables que puedan reinsertarlas a la sociedad.

Se recomienda que exista mayor enfasis en la orientacion sobre la educacion sexual para la zona rural de Yaguachi, creando estrategias para evitar el embarazo precoz y no deseado tanto desde el sector de la educacion como el de la salud, ya que estas jovenes se encuentran en sus años de secundaria y su condicion de estudiante, la educacion sexual podria llegar a ser una herramienta mas estrategica si se hace en el aula de clases. En el caso de la participacion del sector salud, la implicacion del personal de enfermeria radica en poder realizar visitas domiciliarias que permitan identificar las adolescentes del canton Yaguachi y asi crear proyectos especificos de actuacion para la prevencion de las complicaciones posibles del embarazo. Solo un esfuerzo mancomunado del sector publico de la salud, obtendría resultados plausibles para esta poblacion.

\section{Conflicto de intereses}

Ninguno declarado por los autores.

\section{Financiación}

Autofinanciado.

\section{Agradecimientos}

\section{Ninguno declarado por los autores}

\section{Referencias}

1. Casas J, Chumbe O, Inguil R, Gonzales L, Mercado F. El parto en la adolescente. Estudio de 3 grupos adolescencia temprana, tardía y grupo control. Rev Peru Ginecol Obstet. 19 de junio de 2015;44(2):101-6.

2. Sáez Cantero Viviana. Embarazo y Adolescencia: Resultados Perinatales. Rev Cubana Obstet Ginecol [Internet]. 2005 Ago [citado 2018 Feb 24] ; 31(2). Disponible en: http://scielo.sld.cu/scielo.php?script=sci_arttext\&pid=S0138$600 \times 2005000200001 \& \operatorname{lng}=\mathrm{es}$.

3. Romero Aycho L. Amenaza de parto pre termino. Grupo Educativo Universidad Privada de Ica [Internet]. septiembre de 2015 [citado 8 de febrero de 2018]; Disponible en: http://repositorio.upica.edu.pe/xmlui/handle/123456789/45
4. Paranjothy S, Broughton H, Adappa R, Fone D. Teenage pregnancy: who suffers? Arch Dis Child. marzo de 2009;94(3):239-45.

5. de Rodríguez Isabel Cluet, Rossell-Pineda María del Rosario, Álvarez de Acosta Thais, Rojas Quintero Ligia. Factores de riesgo asociados a la prematuridad en recién nacidos de madres adolescentes. Rev Obstet Ginecol Ven [Internet]. 2013 Sep [citado 2018 Feb 25] ; 73( 3 ): 157-170. Disponible en: http://www.scielo.org.ve/scielo.php?script=sci_arttext\&pid=S004 8-77322013000300003\&lng=es.

6. Laterra C, Andina E, Di Marco I. Guía de prácticas clínicas. Amenaza de parto prematuro. Revista del Hospital Materno Infantil Ramón Sardá [Internet]. 2003 [citado 8 de febrero de 2018];22(1). Disponible http://www.redalyc.org/resumen.oa?id=91222107

7. Aristizábal Hoyos, GP, MarleneBlanco Borjas, D, Sánchez Ramos, A, Ostiguín Meléndez, RM. El modelo de promoción de la salud de Nola Pender. Una reflexión en torno a su comprensión. Enfermería Universitaria [Internet]. 2011;8(4):1623. http://www.redalyc.org/articulo.oa?id=358741840003

8.

9. Gavino Gavilanes AG, Sánchez Alvarado AP. Los grupos de atención prioritaria y su relación en la intervención Comunitaria Universitaria: Caso Yaguachi Nuevo. Repositorio de la Universidad Estatal de Milagro [Internet]. septiembre de 2017 [citado 16 de febrero de 2018]; Disponible en: http://repositorio.unemi.edu.ec//handle/123456789/3754

10. Sosa JLC, Flores JLS. Factores de riesgo para parto pretérmino en gestantes del Hospital Provincial Docente Belen de Lambayeque. Rev Cient Sal \& Vid Sip. 15 de noviembre de 2015;2(2):7-18.

11. Vance ME, Keele B. Development and Validation of the Cervical Cancer Knowledge and Beliefs of Appalachian Women Questionnaire. Journal of Nursing Measurement; New York. 2013;21(3):477-501.

12. Aquino $R$, Isabel $G$. Rol de la enfermera en la promoción de estilos de vida saludables aplicando la teoría de nola pender en el Colegio Jorge Cieza Lachos Sjl-Lima 2014. Repositorio Institucional - UAP [Internet]. 2017 [citado 9 de febrero de 2018]; Disponible en: http://repositorio.uap.edu.pe/handle/uap/5612

13. Vázquez Márquez Á, Guerra Verdecia C, Herrera Vicente V de la Cruz Cháve F, Chávez A, M Á. Embarazo y adolescencia: Factores biológicos materno y perinatal más frecuentes. Rev Cubana Obstet Ginecol [Internet]. 2001 Ago [citado 2018 Abr 24] ; 27( 2 ): 158-164. Disponible en: http://scielo.sld.cu/scielo.php?script=sci arttext\&pid=S0138$600 \times 2001000200012 \&$ lng $=$ es.

14. Aspectos sociales del embarazo y la fecundidad adolescente en América Latina | Repositorio Digital | Comisión Económica para América Latina y el Caribe [Internet]. [citado 9 de febrero de 2018]. Disponible http://repositorio.cepal.org/handle/11362/6737

15. Maddaleno M, Morello P, Infante-Espínola F. Salud y desarrollo de adolescentes y jóvenes en Latinoamérica y El Caribe: desafíos para la próxima década. Salud Pública Mex. 2003; 45 (1):S132-S139

16. Borrás Santisteban T. Adolescencia: definición, vulnerabilidad y oportunidad. Correo Científico Médico. marzo de 2014;18(1):05-

17. Ovalle Alfredo, Kakarieka Elena, Rencoret Gustavo, Fuentes Ariel, del Río María José, Morong Carla et al. Factores asociados con el parto prematuro entre 22 y 34 semanas en un hospital público de Santiago. Rev. méd. Chile [Internet]. 2012 Ene [citado 2018 Abr 25] ; 140( 1 ): 19-29. Disponible en:

https://scielo.conicyt.cl/scielo.php?script=sci_arttext\&pid=S003498872012000100003\&lng=es. http://dx.doi.org/10.4067/S003498872012000100003

18. Flores G, Carlos L. "Determinación de los principales factores de riesgo maternos en relación a la ruptura prematura de membranas en pacientes atendidas en el hospital provincial docente ambato periodo enero 2011-enero 2012." 29 de mayo de 2013 [citado 9 de febrero de 2018]; Disponible en: http://repositorio.uta.edu.ec/jspui/handle/123456789/3692

19. Harger JH, Hsing AW, Tuomala RE, Gibbs RS, Mead PB Eschenbach DA, et al. Risk factors for preterm premature rupture of fetal membranes: a multicenter case-control study. Am J Obstet Gynecol. julio de 1990;163(1 Pt 1):130-7.

20. Marvin-Dowle K, Kilner K, Burley VJ, Soltani H. Impact of adolescent age on maternal and neonatal outcomes in the Born in Bradford cohort. BMJ Open. 1 de marzo de 2018;8(3):e016258.

21. Vega J, Astudillo A, Aragundi C, Flores J. Infección de vías urinarias como factor de riesgo de amenaza de parto pretérmino en gestantes adolescentes. RECIMUNDO. 26 de septiembre de 2017;1(4):791-802.

22. Gualpa M, Ricardo L. Morbi - mortalidad por trastornos respiratorios en recién nacidos por cesáreas iterativas en el Hospital Provincial Docente Ambato, durante el período 
diciembre 2012 a mayo 2013. 25 de febrero de 2014 [citado 9 de febrero 2018]; Disponible

http://repositorio.uta.edu.ec/jspui/handle/123456789/6599
Intriago C, Patricia S. El embarazo en adolescentes como factor de riesgo asociado al incremento de la morbilidad neonatal en pacientes que acudieron al servicio de obstetricia de la Clínica San Sebastián de Ambato de Enero a Diciembre 2013. 2015 [citado 9 de febrero de 2018]; Disponible en: http://www.dspace.uce.edu.ec/handle/25000/4667

24. Martín GM, Román ICP. Mortalidad Materna en Pinar del Río, 1991-2011. Revista Cubana de Medicina Intensiva y Emergencias [en línea]. 3 de noviembre de 2014 [27 de enero de 2017];13(3). Disponible en: http://www.revmie.sld.cu/index.php/mie/article/view/33 